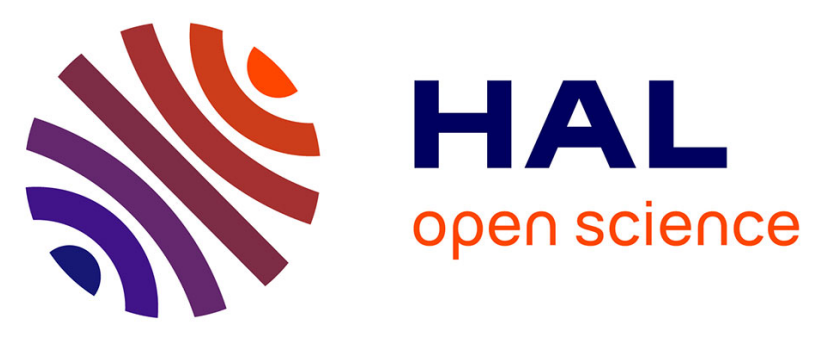

\title{
Photoinduced Chromophore Hydration in the Fluorescent Protein Dreiklang Is Triggered by Ultrafast Excited-State Proton Transfer Coupled to a Low-Frequency Vibration
}

Fabien Lacombat, Pascal Plaza, Marie-Aude Plamont, Agathe Espagne

\section{To cite this version:}

Fabien Lacombat, Pascal Plaza, Marie-Aude Plamont, Agathe Espagne. Photoinduced Chromophore Hydration in the Fluorescent Protein Dreiklang Is Triggered by Ultrafast Excited-State Proton Transfer Coupled to a Low-Frequency Vibration. Journal of Physical Chemistry Letters, 2017, 8 (7), pp.1489-1495. 10.1021/acs.jpclett.7b00348 . hal-03500004

\author{
HAL Id: hal-03500004 \\ https://hal.science/hal-03500004
}

Submitted on 21 Dec 2021

HAL is a multi-disciplinary open access archive for the deposit and dissemination of scientific research documents, whether they are published or not. The documents may come from teaching and research institutions in France or abroad, or from public or private research centers.
L'archive ouverte pluridisciplinaire HAL, est destinée au dépôt et à la diffusion de documents scientifiques de niveau recherche, publiés ou non, émanant des établissements d'enseignement et de recherche français ou étrangers, des laboratoires publics ou privés. 
Photoinduced Chromophore Hydration in the Fluorescent Protein Dreiklang Is Triggered by Ultrafast Excited-State Proton Transfer Coupled to a Low-Frequency Vibration

Fabien Lacombat, ${ }^{\dagger, \ddagger}$ Pascal Plaza, ${ }^{\dagger, \star,}$ Marie-Aude Plamont ${ }^{\dagger, \star, \S}$ and Agathe Espagne ${ }^{*, \dagger, \star, \S}$

\author{
${ }^{\dagger}$ Ecole normale supérieure, PSL Research University, Département de Chimie, PASTEUR, 24 rue \\ Lhomond, 75005 Paris, France \\ ${ }^{\ddagger}$ Sorbonne Universités, UPMC Univ Paris 06, PASTEUR, 24 rue Lhomond, 75005 Paris, France \\ ${ }^{\S}$ CNRS, PASTEUR, 24 rue Lhomond, 75005 Paris, France
}

\title{
Corresponding author:
}

agathe.espagne@ens.fr 


\begin{abstract}
:
Due to growing applications in advanced fluorescence imaging, the mechanisms and dynamics of photoinduced reactions in reversibly photoswitchable fluorescent proteins are currently attracting much interest. We report the first time-resolved study of the photoswitching of Dreiklang, so far the only fluorescent protein to undergo reversible photoinduced chromophore hydration. Using broadband femtosecond transient absorption spectroscopy, we show that the reaction is triggered by an ultrafast deprotonation of the chromophore phenol group in the excited state, in $100 \mathrm{fs}$. This primary step is accompanied by coherent oscillations that we assign to its coupling with a low-frequency mode, possibly a deformation of the chromophore hydrogen bond network. A ground-state intermediate is formed in the ps-ns regime, that we tentatively assign to the deprotonated water adduct. We suggest that proton ejection from the phenol group leads to a charge transfer from the phenol to the imidazolinone ring, which triggers imidazolinone protonation by nearby Glu222 and catalyzes the addition of the water molecule.
\end{abstract}

\title{
Table of Contents Graphic :
}

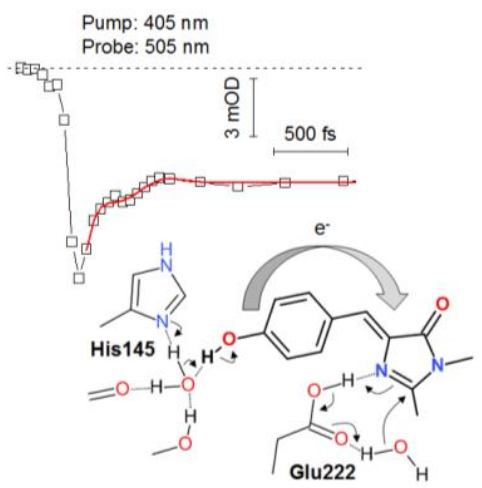


Reversibly photoswitchable fluorescent proteins (RSFPs) are Green Fluorescent Protein (GFP) homologues that can be switched back and forth between a fluorescent (ON) state and a nonfluorescent (OFF) state by irradiation at two wavelengths. Over the last decade, they have become essential tools for super-resolution microscopy and other advanced fluorescence imaging techniques. ${ }^{1} \mathrm{X}$-ray crystallography studies of the ON and OFF states of several RSFPs revealed that in most cases, photoswitching is based on cis/trans isomerization of the chromophore coupled with proton transfer. ${ }^{2-4}$ The photoswitching pathways of this class of RSFPs, the prototype of which is Dronpa, ${ }^{5}$ have been further investigated by time-resolved spectroscopy with visible $^{6-9}$ and $\mathrm{IR}^{10,11}$ probes, quantum chemistry ${ }^{12,13}$ and low-temperature X-ray crystallography, ${ }^{14}$ in order to establish the sequence in time of the two events, their characteristic timescales and detailed mechanisms. Although questions remain, a clear picture of chromophore transformations involved in the $\mathrm{OFF} \rightarrow \mathrm{ON}$ photoswitching of Dronpa has recently emerged: the first step consists of picosecond trans $\rightarrow$ cis isomerization of the chromophore in the excited-state, while proton transfer follows much later in the ground state, on the microsecond timescale. ${ }^{7,11}$

In Dronpa-like RSFPs, the wavelength used to excite and image the fluorescence of the ON state under a microscope is identical to one of the wavelengths driving the ON-OFF transitions (e.g. $\mathrm{ON} \rightarrow \mathrm{OFF}$ for Dronpa ${ }^{5}$ and $\mathrm{OFF} \rightarrow \mathrm{ON}_{\text {for }} \mathrm{Padron}^{4}$ ), which prevents a perfect control of the ON or OFF state of the probe during data acquisition. Brakemann et al. recently tackled this problem by developing Dreiklang, ${ }^{15}$ a green-emitting RSFP in which fluorescence excitation is decoupled from photoswitching. Interestingly, X-ray crystallography combined with mass spectrometry ${ }^{15}$ showed that Dreiklang exhibits an entirely new photoswitching mechanism, based on the reversible covalent addition of a water molecule across the $\mathrm{C}=\mathrm{N}$ bond of the chromophore imidazolinone ring, as illustrated in Scheme 1. The reaction reduces the electronic delocalization within the chromophore, causing a strong blue-shift of the absorption of the OFF state, ${ }^{15}$ which is 
key for disentangling fluorescence from photoswitching. Dreiklang is still today the only reported example of a fluorescent protein displaying such photochemistry. The thermal formation of a hydrated structure similar to Dreiklang's OFF-state has however been proposed to occur in some fluorescent proteins during chromophore maturation, ${ }^{16-18}$ suggesting that photoinduced hydration might be transferrable to other members of the GFP family. In spite of its originality and high potential for the development of advanced RSFPs, the dynamics and mechanistic details of Dreiklang photoswitching are still completely unknown. We report here the first time-resolved study of Dreiklang photoswitching. The ON $\rightarrow$ OFF photoconversion was investigated from $\sim 100$ fs to $10 \mathrm{~ns}$ using broadband femtosecond UV-visible transient absorption (TA) spectroscopy, in order to establish the events immediately following the absorption of a photon and the onset of the hydration process.

Figure 1 shows the absorption spectra of Dreiklang at $\mathrm{pH} 7.5$, in the equilibrium ON state and after irradiation at $\sim 410 \mathrm{~nm}$ (in agreement with the report of Brakemann et al. ${ }^{15}$ ). In the ON state (black line), the two bands peaking at $413 \mathrm{~nm}$ and $511 \mathrm{~nm}$ correspond to the neutral (A) and anionic (B) forms of the chromophore, respectively. ${ }^{15}$ Irradiation in the A band switches the protein to the OFF state, characterized by a strongly blue-shifted band, peaking at $339 \mathrm{~nm}(\mathrm{C}$; Figure 1, red line). The ON state can be regenerated by irradiation of the OFF state in the $\mathrm{C}$ band. It also recovers spontaneously in the dark with a half-life of $\sim 10 \mathrm{~min}$ at room temperature, which explains the residual A and B bands observed after 410-nm irradiation. Excitation of the B band (ON state) elicits a strong green fluorescence with a maximum at $525 \mathrm{~nm}$ (Figure 2A, grey dashdotted line). Interestingly, the weak steady-state fluorescence spectrum of form A is identical to that of form B (see Supporting Information (SI), Figure S4), which suggests that the phenol group of the neutral chromophore deprotonates very fast in the excited state. 
Form A (ON state) was excited at $405 \mathrm{~nm}$ with $110-\mathrm{fs}$ pulses, to initiate the $\mathrm{ON} \rightarrow \mathrm{OFF}$ switching. The experiment was performed at $\mathrm{pH} 6.3$ in order to increase the amount of neutral chromophore $\left(\mathrm{pK}_{\mathrm{a}}=7.2 ;{ }^{15}\right.$ Figure S3). Homologous measurements were performed in $\mathrm{D}_{2} \mathrm{O}$ (Figures S1 and S2) to characterize proton transfer steps. Photoinduced spectral changes were recorded between 300 and $700 \mathrm{~nm}$, as shown in Figure 2. The initial spectrum at $100 \mathrm{fs}$ (black line in Figure 2A) exhibits several characteristic bands. The negative one extending from 360 to $430 \mathrm{~nm}$ matches the steady-state absorption of form A (grey dotted line) and is attributed to ground-state bleaching (GSB). The other negative band, ranging from 465 to $630 \mathrm{~nm}$, is due to stimulated emission (SE). The shoulder at $\sim 560 \mathrm{~nm}$ evokes the fluorescence spectrum of form B (grey dash-dotted line) and suggests that the red wing of the band includes the contribution of some readily deprotonated population. The blue wing (below $490 \mathrm{~nm}$ ) has however no equivalent in the fluorescence spectrum of form B. It proves that a population of excited neutral chromophores (noted $\mathrm{A}^{*}$ ) is also present at $100 \mathrm{fs}$. GFP-like fluorescent proteins containing neutral chromophores have indeed been reported to emit in the 400 to $600 \mathrm{~nm}$ spectral range, with maxima at $470-480 \mathrm{~nm} \cdot{ }^{7,19-21} \mathrm{We}$ show as an example the fluorescence spectrum of the $\mathrm{A}^{*}$ state of GFP, measured by Jaye et al. ${ }^{21}$ before excited-state deprotonation of the chromophore, by time-resolved fluorescence spectroscopy (grey dashed line). Finally, the three positive bands (below $360 \mathrm{~nm}$, from 430 to $465 \mathrm{~nm}$ and above $630 \mathrm{~nm}$ ) correspond to spectral regions where excited-state absorption (ESA) dominates the other contributions. Let us note that a significant contribution of SE from $\mathrm{A}^{*}$ is expected in the region of the central ESA band.

The transient spectra evolve then in three steps, highlighted in sections A-C of Figure 2. During the first $500 \mathrm{fs}$ (Figure 2A), one observes mainly a rise of the central ESA band, a decay of SE below $514 \mathrm{~nm}$ and a rise of SE emission above $514 \mathrm{~nm}$ (with temporary isosbestic point at $514 \mathrm{~nm})$. We assign these spectral changes to the deprotonation of $\mathrm{A}^{*}$, leading to an excited 
anionic species noted I*. At 500 fs (blue spectrum), the characteristic SE of A* below $490 \mathrm{~nm}$ has indeed entirely disappeared and the new SE band peaks at $523 \mathrm{~nm}$, close to the fluorescence maximum of form $\mathrm{B}$. The inset of Figure $2 \mathrm{~A}$ shows the double difference spectrum $\Delta \Delta \mathrm{A}=$ $\Delta \mathrm{A}(500 \mathrm{fs})-\Delta \mathrm{A}(100 \mathrm{fs})$ between 440 and $580 \mathrm{~nm}$ (black line), which illustrates the early changes. We could satisfactorily simulate $\Delta \Delta \mathrm{A}$ by a weighted difference of anionic and neutral SE (inset of Figure 2A, red line; see Methods in SI for details). This demonstrates that the rise of the central ESA band around $460 \mathrm{~nm}$ is mainly due to the disappearance of the underlying SE of $A^{*}$. GSB shows little evolution on this ultrashort timescale, suggesting a high yield of $A^{*}$ deprotonation. Finally, the partial decays of ESA below $360 \mathrm{~nm}$ and above $630 \mathrm{~nm}$ point to a lower absorption of $\mathrm{I}^{*}$ in these regions, compared to $\mathrm{A}^{*}$. It is well-known that an excited-state proton transfer (ESPT) also occurs in GFP upon excitation of its neutral form, in a few picoseconds. $^{22,23}$ Remarkably, the dynamics is here more than one order of magnitude faster. The spectral signature is however qualitatively similar. ${ }^{22}$

From 500 fs to 3 ps (Figure 2B), the ESA and GSB bands below $490 \mathrm{~nm}$ decay significantly, while the SE band remains essentially stable. To rationalize these apparently asynchronous evolutions of ESA and SE from the same species, we hypothesized that a slight reshaping of the ESA band was "amplified" by the overlap with GSB. The contribution of GSB was estimated by reference to the TA signal of a solution of $\left[\mathrm{Ru}(\mathrm{bpy})_{3}\right]^{2+}$ (see Methods in SI for more details), and subtracted from the data. GSB-free spectra are displayed in the inset of Figure 2B. They indeed show a slight narrowing and blue-shift of the ESA band, in line with the slight narrowing of SE (Figure 2B, main frame). We assign these spectral changes to the vibrational cooling of I*.

Finally, the ps-to-ns timescale (Figure 2C) is characterized by a simultaneous decay of all bands, reflecting the decay of $\mathrm{I}^{*}$. Excited-state contributions are still present at $2.6 \mathrm{~ns}$ (pink spectrum), as evidenced by the remaining SE, and obscure the detection of potential ground-state 
photoproducts. An additional TA spectrum recorded at $10 \mathrm{~ns}$ (inset of Figure 2C) is however fully devoid of SE, showing that the decay of $\mathrm{I}^{*}$ is complete at this delay. The spectrum consists of GSB and of the absorption band of a ground-state photoproduct (apparent maximum at 450 $\mathrm{nm}$ ), which we named $\mathrm{X}$. The signal is zero below $365 \mathrm{~nm}$, where OFF-state absorption is expected (see Figure 1). We conclude that the switching reaction is not over at $10 \mathrm{~ns}$.

A major result at this stage is that the $\mathrm{ON} \rightarrow \mathrm{OFF}$ photoconversion of Dreiklang involves as intermediate an excited anionic species $\left(\mathrm{I}^{*}\right)$ formed by excited-state deprotonation of form A. In these circumstances, one may wonder why the OFF state cannot be produced by direct excitation of the anionic B form. To clarify this point, we measured the spectral changes triggered by femtosecond excitation (80-fs pulses) of form B at 505 nm, at pH 10.0 (see Figure S3). As can be seen in Figure 3, the recorded TA spectra are made up of two bands: a positive ESA band below $465 \mathrm{~nm}$ and a negative band from $465 \mathrm{~nm}$ to $\sim 650 \mathrm{~nm}$, resulting from overlapping GSB and SE contributions (see the grey dotted steady-state absorption and fluorescence spectra). They decay simultaneously on the nanosecond timescale, as the excited state (noted $\mathrm{B}^{*}$ ) decays. Comparison of Figure $2 \mathrm{C}$ with Figure 3 shows that the lifetime of $\mathrm{I}^{*}$ is much shorter than that of $\mathrm{B}^{*}$. The TA signal half life is $\sim 30 \mathrm{ps}$ in the first case $\left(\mathrm{I}^{*}\right)$, and $\sim 1 \mathrm{~ns}$ in the second case $\left(\mathrm{B}^{*}\right)$, thereby demonstrating that the two species are different. Moreover, the TA spectrum measured $20 \mathrm{~ns}$ after excitation of B shows no significant absorption around $450 \mathrm{~nm}$ (inset of Figure 3; the small negative signal seen at $\sim 515 \mathrm{~nm}$ is likely due to some minor long component of the excited-state decay). This is in sharp contrast with the formation of $\mathrm{X}$ upon $\mathrm{I}^{*}$ decay (inset of Figure $2 \mathrm{C}$ ). We suggest that the peculiar reactivity of species $\mathrm{I}^{*}$ is due to the fact that it combines the charge distribution of the excited anionic chromophore with the protein conformation of the neutral ground state. 
We next proceeded to kinetic analysis of the TA data by a standard multiexponential global fitting procedure. Inspection of the TA kinetics of both the A and B forms however revealed lowfrequency oscillations occurring during the first picosecond, as shown at representative wavelengths in Figure 4, panels $\mathrm{A}$ and $\mathrm{C}$. The modulation is particularly obvious at $520 \mathrm{~nm}$ for form B (Figure 4C, green line) and at $505 \mathrm{~nm}$ for form A (Figure 4A, dark blue trace). Oscillations are also observed in the TA kinetics of form $\mathrm{A}$ in $\mathrm{D}_{2} \mathrm{O}$ (Figure $\mathrm{S} 2 \mathrm{~A}$ ). These oscillations were taken into account by adding a damped cosine with wavelength-dependent phase to our fit function (see Methods in SI for details). The frequencies, periods and damping times of the oscillatory components of the different samples are given in Table 1 and their amplitude and phase spectra are shown in the insets of Figures 4B, 4D and S2B (amplitude in black, phase in red). The insets of Figures 4A and S2A display the oscillatory parts of the fits for form $\mathrm{A}$ in $\mathrm{H}_{2} \mathrm{O}$ and $\mathrm{D}_{2} \mathrm{O}$.

The strong wavelength-dependence of the phases points to coherent oscillations resulting from the excitation of vibrational wave packets by the spectrally broad femtosecond pump pulses. ${ }^{24-26}$ Vibrational wave packets can be created in the excited state, but also in the ground state, through impulsive stimulated Raman scattering. ${ }^{27}$ Ground-state wave packets modulate GSB, while excited-state wave packets modulate ESA and SE. Photoproduct bands may also be concerned, when the vibrational coherence persists throughout a photochemical reaction. ${ }^{26,28}$ The affected band shifts back and forth as a result of wave packet motion about the corresponding excited- or ground-state potential energy surface (PES) minimum, leading to out-of-phase oscillations of the kinetics in its blue and red sides. ${ }^{25,27}$ When a single electronic transition is probed, the maximum of the band is expected to feature both a minimum in the amplitude of the modulation and a phase jump of $\pi .^{25,27}$ The oscillatory pattern may however get more complicated when several contributions overlap. 
The oscillations in the TA kinetics of form B are mainly localized in the $480-550 \mathrm{~nm}$ region, where both GSB and SE contribute (inset of Figure 4D). Their amplitude goes through a minimum and their phase jumps simultaneously by $\sim \pi$ at $525 \mathrm{~nm}$, the maximum of the SE contribution (see steady-state fluorescence spectrum in Figure 3). We conclude that the observed modulation originates mainly from vibrational wave packet motion on the excited-state PES. In the case of form A, the oscillatory behavior is observed over a broad spectral range extending from 350 to $600 \mathrm{~nm}$ (inset of Figure 4B). Since GSB does not contribute above $480 \mathrm{~nm}$ (see steady-state absorption spectrum in Figure 2A), the oscillations in the red part of the TA spectrum indicate that an excited-state wave packet is again involved. The amplitude of the modulation is the highest between 425 and $525 \mathrm{~nm}$, the region where the main spectral changes associated to the ESPT reaction are observed (Figure 2A). Interestingly, a sharp phase jump of $\sim \pi$ and a minimum in the amplitude are seen at $525 \mathrm{~nm}$, peak of the anionic SE, but not at $475 \mathrm{~nm}$, peak of the neutral SE. ${ }^{7,19-21}$ We propose that the ESPT step is too fast compared to the 630-fs vibration period (see Table 1 ) to allow the wave packet to oscillate in the reactant well $\left(\mathrm{A}^{*}\right.$ species), but that the vibrational coherence persists throughout the reaction and is observed in the longer-lived photoproduct I* This scenario is supported by the kinetic analysis, which sets the time constant for ESPT to 100 fs (see below). The $\sim \pi$ phase jump and amplitude minimum found at $425 \mathrm{~nm}$ could then originate from oscillations in the ESA contribution of $\mathrm{I}^{*}$, expected in this very region (see the inset of Figure $2 \mathrm{~B}$ ).

Similar low-frequency oscillations have been observed in the femtosecond transient grating and stimulated Raman data of $\mathrm{GFP}^{29}$ and one of its mutants ${ }^{30}$, albeit with higher frequencies of 95 and $120 \mathrm{~cm}^{-1}$. They have been assigned to the coherent excitation of intramolecular deformation modes of the excited chromophore. ${ }^{29,30}$ The lower frequencies of the oscillations observed here in the femtosecond TA data of Dreiklang $\left(30-90 \mathrm{~cm}^{-1}\right.$; see Table 1) suggest that they are rather due 
to the excitation of intermolecular modes involving the chromophore and its environment. Moreover, the sensitivity of the low-frequency mode of form A to deuteration $\left(31 \mathrm{~cm}^{-1}\right.$ in $\mathrm{D}_{2} \mathrm{O}$ compared to $53 \mathrm{~cm}^{-1}$ in $\mathrm{H}_{2} \mathrm{O}$ ) suggests that it involves proton motion. One could think of a mode implying a deformation of the hydrogen bond network around the chromophore. The excited electronic transition, of charge transfer character, has indeed been reported to involve a decrease of the electron density on the phenol oxygen, ${ }^{29,31}$ which is expected to displace the equilibrium geometry of the hydrogen bond network in which it participates.

In addition to the oscillatory component, three independent exponentials plus a biexponential decay (i.e. fixed ratio of two exponentials with a single amplitude spectrum) were needed to achieve a good fit of the TA data of form A, while a biexponential decay was sufficient to fit those of form B. The time constants are gathered in Table 1, and the corresponding amplitude spectra, or DADS (decay associated difference spectra) are shown in Figures 4B, 4D and S2B.

The 100-fs component of form A (Table 1) corresponds to ESPT, which is hence 50 times faster than in neutral GFP. ${ }^{22,23}$ As previously mentioned, this lifetime is much shorter than the 630-fs period of the coherent oscillations, which explains why the vibrational coherence is mainly observed in $I^{*}$. Moreover, the ESPT dynamics in Dreiklang is significantly less sensitive to H/D exchange than in GFP (slowing down by a factor of 1.5 instead of $\sim 5^{22,23}$; Table 1 ). Interestingly, the isotope effect is comparable to that on the period of the low-frequency vibration (slowing down by a factor of 1.7), suggesting that both phenomena are intimately related. We tentatively propose that ESPT in Dreiklang is gated by the coherently excited low-frequency vibration, which allows the protein to reach, after $100 \mathrm{fs}$, a nuclear configuration much more favorable to proton transfer than the Franck-Condon configuration. This idea is illustrated in the contour diagram of the excited-state PES at the top of Scheme 2A. 
The 750-fs lifetime of form A (1.3 ps in $\mathrm{D}_{2} \mathrm{O}$; Table 1) is assigned to the vibrational cooling of $\mathrm{I}^{*}$, and the three longer lifetimes (19 ps, $108 \mathrm{ps}$ and $1.8 \mathrm{~ns}$ ) to its multiexponential decay to $\mathrm{X}$ (Scheme 2A). Multiexponential kinetics in proteins are often attributed to structural heterogeneity. ${ }^{7,8,10,32}$ The X-ray structure of ON-state Dreiklang at $100 \mathrm{~K}$ shows only a single conformation, ${ }^{15}$ but the three decay times could be due to some conformational flexibility of the protein at room temperature, on timescales longer than the probed dynamics, as has been reported for other GFP variants. ${ }^{33}$ The fact that two of the decay components can be fitted with a single biexponential decay is in agreement with this interpretation. No satisfactory fit could however be obtained replacing all three decay components by a triexponential decay, presumably because the 19-ps component still reflects some vibrational cooling of $I^{*}$ mixed to the excited-state decay, as suggested by its comparatively larger amplitude in the 350-500 $\mathrm{nm}$ region (Figure 4B). These fits confirm that the lifetime of $\mathrm{I}^{*}$ is much shorter than that of the anion formed by direct excitation: the average lifetimes at $525 \mathrm{~nm}$ are $219 \mathrm{ps}\left(\mathrm{I}^{*}\right)$ and $2.3 \mathrm{~ns}\left(\mathrm{~A}^{*}\right)$. In addition, H/D exchange results in a slowing down of $\mathrm{I}^{*}$ decay by a factor of $\sim 2$ (average lifetime of $469 \mathrm{ps}$ in $\mathrm{D}_{2} \mathrm{O}$ ), suggesting the involvement of proton motion in the quenching mechanism.

The present work establishes ultrafast excited-state deprotonation of the chromophore phenol group as the primary step of Dreiklang's $\mathrm{ON} \rightarrow \mathrm{OFF}$ photoconversion. The connection with the final addition of a water molecule to the imidazolinone ring is however not obvious. We give below a few directions. Inspection of the ON-state X-ray structure in the vicinity of the chromophore hydroxyl shows that it is hydrogen-bonded to a water molecule, which itself interacts with a histidine residue (His145; Scheme 2B). We propose that the photoejected proton is accepted by this water molecule, which in turn protonates His145 (Scheme 2B, step 1). Deprotonation is then expected to trigger a charge redistribution in the chromophore, leading to an increase of the electron density on the imidazolinone ring (Scheme 2B, step 2). This is due to 
the $\pi$-conjugated and electron donor-acceptor nature of the chromophore structure. Interestingly, the hydrogen-bond network on the imidazolinone side comprises a glutamic acid residue (Glu222) which is suitably located to catalyze the addition of the water molecule (Scheme 2B). Mutagenesis studies by Brakemann et al. ${ }^{15}$ suggest that Glu222 is crucial for the photoswitching properties of Dreiklang. We propose that Glu222 is protonated in form A and transfers its proton to nitrogen $\mathrm{N}_{\mathrm{a}}$ in response to the increase in electron density on the imidazolinone ring (Scheme $2 B$, step 3). Protonation of $N_{a}$ would indeed activate the $C_{b}=N_{a}$ bond and presumably catalyze the nucleophilic addition of the water molecule, which is only $3.5 \AA$ away from electrophilic $\mathrm{C}_{\mathrm{b}}$. Glu222 could in addition increase the nucleophilicity of the water molecule by abstracting a proton from it.

The fact that $\mathrm{X}$ is formed following excitation of the A state, but not of the B state suggests that it plays the role of an intermediate in the photoswitching reaction. Within the above framework, one could imagine that $\mathrm{X}$ corresponds to the zwitterionic species formed upon imidazolinone protonation. This hypothesis seems however unlikely, because quantum chemistry calculations carried out on RSFPs predict that the chromophore zwitterion absorbs at longer wavelengths than the anion. ${ }^{34,35}$ This has also been experimentally verified in the case of a methylated derivative of the GFP chromophore in solution. ${ }^{36} \mathrm{X}$ could more likely be the anionic water adduct formed from $\mathrm{I}^{*}$ by the concerted mechanism illustrated in Scheme 2B (step 3). Further evolution of $\mathrm{X}$ to the final OFF state would then involve phenolate protonation and conformational relaxations of the chromophore and its environment. This would be in line with the strongly blue-shifted absorption of the OFF state, since phenolate protonation is expected to reduce electronic delocalization within the chromophore.

In conclusion, we studied the photoswitching dynamics of Dreiklang for the first time by timeresolved spectroscopy. We found that the $\mathrm{ON} \rightarrow \mathrm{OFF}$ photoconversion, which ultimately results in 
chromophore hydration, ${ }^{15}$ is triggered by an ultrafast deprotonation of the chromophore phenol group in the excited state, with a time constant of $100 \mathrm{fs}$. This primary reaction is accompanied by coherent oscillations that we assign to the coupling of the reactive motion with a lowfrequency mode, possibly a deformation of the chromophore hydrogen bond network. Decay of the deprotonated species $\left(I^{*}\right)$ on ps-ns time scales leads to the formation of a ground-state intermediate $(\mathrm{X})$. We suggest that charge transfer from the phenol to the imidazolinone ring in $\mathrm{I}^{*}$ triggers protonation of the $\mathrm{C}=\mathrm{N}$ bond nitrogen by nearby Glu222, thereby catalyzing the addition of the water molecule in $\mathrm{X}$. Conversion of $\mathrm{X}$ to the final OFF state would require phenolate reprotonation, on a timescale longer than our observation window (10 ns).

\section{Acknowledgments}

We acknowledge financial support from CNRS, Ecole Normale Supérieure, P. and M. Curie University and the P.-G. de Gennes foundation (grant FPGG 033).

\section{Supporting Information}

Experimental methods, femtosecond TA data of form $\mathrm{A}$ in $\mathrm{D}_{2} \mathrm{O}$, steady-state absorption spectra of the $\mathrm{ON}$ state at different $\mathrm{pH}$, steady-state fluorescence spectra of forms A and $\mathrm{B}$. This material is available free of charge via the Internet at http://pubs.acs.org.

\section{References}

(1) Zhou, X. X.; Lin, M. Z. Curr. Opin. Chem. Biol. 2013, 17, 682-690.

(2) Andresen, M.; Stiel, A. C.; Trowitzsch, S.; Weber, G.; Eggeling, C.; Wahl, M. C.; Hell, S. W.; Jakobs, S. Proc. Natl. Acad. Sci. U. S. A. 2007, 104, 13005-13009.

(3) Adam, V.; Lelimousin, M.; Boehme, S.; Desfonds, G.; Nienhaus, K.; Field, M. J.; Wiedenmann, J.; McSweeney, S.; Nienhaus, G. U.; Bourgeois, D. Proc. Natl. Acad. Sci. U. S. A. 2008, 105, 18343-18348. 
(4) Brakemann, T.; Weber, G.; Andresen, M.; Groenhof, G.; Stiel, A. C.; Trowitzsch, S.; Eggeling, C.; Grubmuller, H.; Hell, S. W.; Wahl, M. C.et al. J. Biol. Chem. 2010, 285, 14603-14609.

(5) Ando, R.; Mizuno, H.; Miyawaki, A. Science 2004, 306, 1370-1373.

(6) Fron, E.; Flors, C.; Schweitzer, G.; Habuchi, S.; Mizuno, H.; Ando, R.; Schryver, F. C.; Miyawaki, A.; Hofkens, J. J. Am. Chem. Soc. 2007, 129, 4870-4871.

(7) Yadav, D.; Lacombat, F.; Dozova, N.; Rappaport, F.; Plaza, P.; Espagne, A. J. Phys. Chem. B 2015, $119,2404-2414$.

(8) Walter, A.; Andresen, M.; Jakobs, S.; Schroeder, J.; Schwarzer, D. J. Phys. Chem. B 2015, 119, 5136-5144.

(9) Colletier, J. P.; Sliwa, M.; Gallat, F. X.; Sugahara, M.; Guillon, V.; Schiro, G.; Coquelle, N.; Woodhouse, J.; Roux, L.; Gotthard, G.et al. J. Phys. Chem. Lett. 2016, 7, 882-887.

(10) Lukacs, A.; Haigney, A.; Brust, R.; Addison, K.; Towrie, M.; Greetham, G. M.; Jones, G. A.; Miyawaki, A.; Tonge, P. J.; Meech, S. R. J. Phys. Chem. B 2013, 117, 11954-11959.

(11) Warren, M. M.; Kaucikas, M.; Fitzpatrick, A.; Champion, P.; Sage, J. T.; van Thor, J. J. Nat. Commun. 2013, 4, 1461.

(12) Li, X.; Chung, L. W.; Mizuno, H.; Miyawaki, A.; Morokuma, K. J. Phys. Chem. B 2010, 114, 11141126.

(13) Morozov, D.; Groenhof, G. Angew. Chem. Int. Ed. Engl. 2016, 55, 576-578.

(14) Faro, A. R.; Carpentier, P.; Jonasson, G.; Pompidor, G.; Arcizett, D.; Demachy, I.; Bourgeois, D. J. Am. Chem. Soc. 2011, 133, 16362-16365.

(15) Brakemann, T.; Stiel, A. C.; Weber, G.; Andresen, M.; Testa, I.; Grotjohann, T.; Leutenegger, M.; Plessmann, U.; Urlaub, H.; Eggeling, C.et al. Nat. Biotechnol. 2011, 29, 942-947.

(16) Barondeau, D. P.; Kassmann, C. J.; Tainer, J. A.; Getzoff, E. D. Biochemistry 2005, 44, 1960-1970.

(17) Rosenow, M. A.; Huffman, H. A.; Phail, M. E.; Wachter, R. M. Biochemistry 2004, 43, 4464-4472.

(18) Siegbahn, P. E. M.; Wirstam, M.; Zimmer, M. Int. J. Quantum Chem. 2001, 81, 169-186.

(19) McAnaney, T. B.; Shi, X. H.; Abbyad, P.; Jung, H.; Remington, S. J.; Boxer, S. G. Biochemistry 2005, 44, 8701-8711.

(20) Stoner-Ma, D.; Jaye, A. A.; Ronayne, K. L.; Nappa, J.; Tonge, P. J.; Meech, S. R. Chem. Phys. 2008, 350, 193-200.

(21) Jaye, A. A.; Stoner-Ma, D.; Matousek, P.; Towrie, M.; Tonge, P. J.; Meech, S. R. Photochem. Photobiol. 2006, 82, 373-379.

(22) Kennis, J. T.; Larsen, D. S.; van Stokkum, I. H.; Vengris, M.; van Thor, J. J.; van Grondelle, R. Proc. Natl. Acad. Sci. U. S. A. 2004, 101, 17988-17993.

(23) Chattoraj, M.; King, B. A.; Bublitz, G. U.; Boxer, S. G. Proc. Natl. Acad. Sci. U. S. A. 1996, 93, 8362-8367.

(24) Rose, T. S.; Rosker, M. J.; Zewail, A. H. J. Chem. Phys. 1988, 88, 6672-6673.

(25) Vos, M. H.; Rappaport, F.; Lambry, J. C.; Breton, J.; Martin, J. L. Nature 1993, 363, 320-325.

(26) Wang, Q.; Schoenlein, R. W.; Peteanu, L. A.; Mathies, R. A.; Shank, C. V. Science 1994, 266, 422424. 
(27) Pollard, W. T.; Dexheimer, S. L.; Wang, Q.; Peteanu, L. A.; Shank, C. V.; Mathies, R. A. J. Phys. Chem. 1992, 96, 6147-6158.

(28) Leonard, J.; Schapiro, I.; Briand, J.; Fusi, S.; Paccani, R. R.; Olivucci, M.; Haacke, S. Chem. Eur. J. 2012, 18, 15296-15304.

(29) Fang, C.; Frontiera, R. R.; Tran, R.; Mathies, R. A. Nature 2009, 462, 200-205.

(30) Litvinenko, K. L.; Meech, S. R. Phys. Chem. Chem. Phys. 2004, 6, 2012-2014.

(31) Helms, V.; Winstead, C.; Langhoff, P. W. J. Mol. Struct. THEOCHEM 2000, 506, 179-189.

(32) Larsen, D. S.; van Stokkum, I. H.; Vengris, M.; van Der Horst, M. A.; de Weerd, F. L.; Hellingwerf, K. J.; van Grondelle, R. Biophys. J. 2004, 87, 1858-1872.

(33) Seifert, M. H.; Georgescu, J.; Ksiazek, D.; Smialowski, P.; Rehm, T.; Steipe, B.; Holak, T. A. Biochemistry 2003, 42, 2500-2512.

(34) Li, X.; Chung, L. W.; Mizuno, H.; Miyawaki, A.; Morokuma, K. J. Phys. Chem. B 2010, 114, 11141126 .

(35) Schafer, L. V.; Groenhof, G.; Klingen, A. R.; Ullmann, G. M.; Boggio-Pasqua, M.; Robb, M. A.; Grubmuller, H. Angew. Chem. Int. Ed. Engl. 2007, 46, 530-536.

(36) Dong, J.; Solntsev, K. M.; Tolbert, L. M. J. Am. Chem. Soc. 2006, 128, 12038-12039. 
Table 1: Global Kinetic Analysis of the Transient Absorption Data of ON State Dreiklang in its Neutral (A) and Anionic (B) Forms. Frequencies, Periods and Damping Times of the Oscillatory Parts. Time Constants of the Multiexponential Parts.

\begin{tabular}{|c|c|c|c|c|c|c|c|c|c|}
\hline & & \multicolumn{3}{|c|}{ Oscillatory part } & \multicolumn{5}{|c|}{ Multiexponential part } \\
\hline & & $v\left(\mathrm{~cm}^{-1}\right)$ & $\mathrm{T}(\mathrm{ps})$ & $\tau_{0}(\mathrm{ps})$ & $\tau_{1}(\mathrm{ps})$ & $\tau_{2}(\mathrm{ps})$ & $\tau_{3}(\mathrm{ps})$ & $\tau_{4}(\mathrm{ps})$ & $\tau_{5}(\mathrm{ps})$ \\
\hline \multirow[t]{2}{*}{ A } & $\mathrm{H}_{2} \mathrm{O}$ & $53 \pm 5$ & $0.63 \pm 0.06$ & $0.24 \pm 0.04$ & $0.10 \pm 0.01$ & $0.75 \pm 0.03$ & $19 \pm 1$ & $108 \pm 8$ & $1800 \pm 200$ \\
\hline & $\mathrm{D}_{2} \mathrm{O}$ & $31 \pm 3$ & $1.1 \pm 0.1$ & $0.29 \pm 0.03$ & $0.15 \pm 0.01$ & $1.3 \pm 0.1$ & $10 \pm 2$ & $144 \pm 14$ & $1100 \pm 90$ \\
\hline $\mathrm{B}$ & $\mathrm{H}_{2} \mathrm{O}$ & $86 \pm 2$ & $0.39 \pm 0.01$ & $0.36 \pm 0.05$ & $368 \pm 61$ & $2700 \pm 450$ & & & \\
\hline
\end{tabular}


Figure 1:

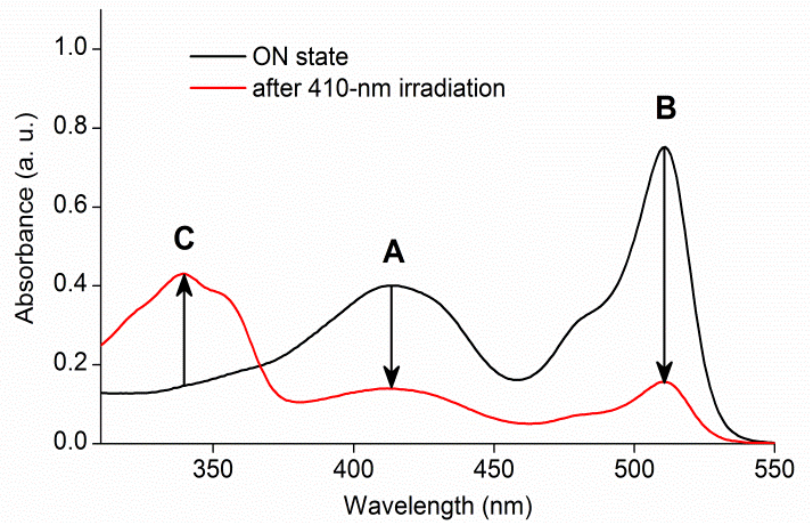


Figure 2:

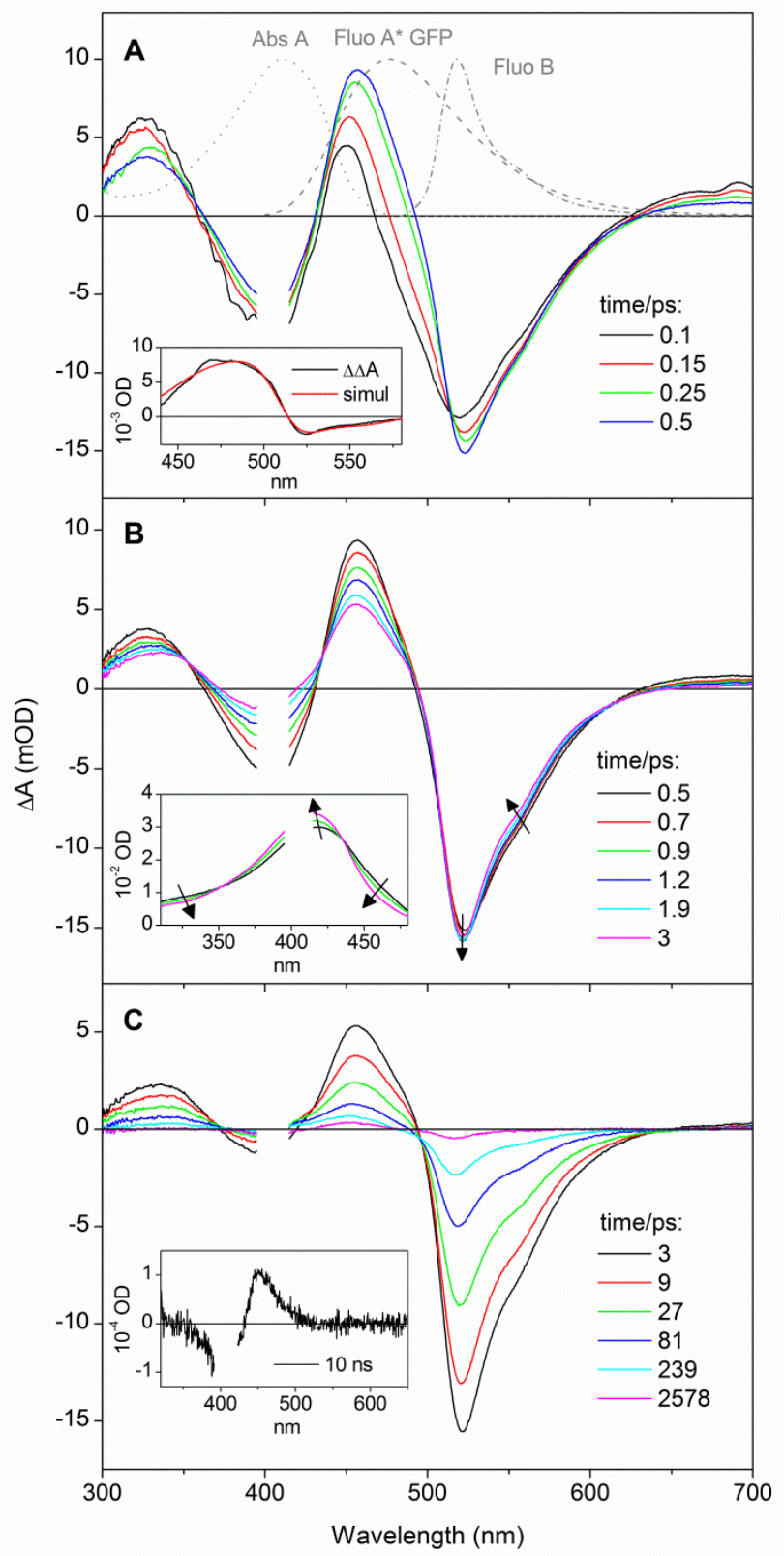


Figure 3:

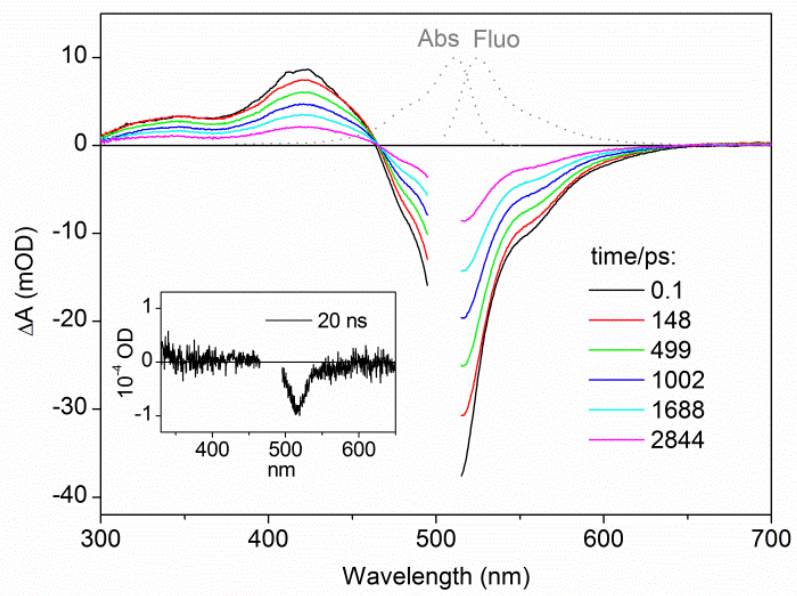


Figure 4:
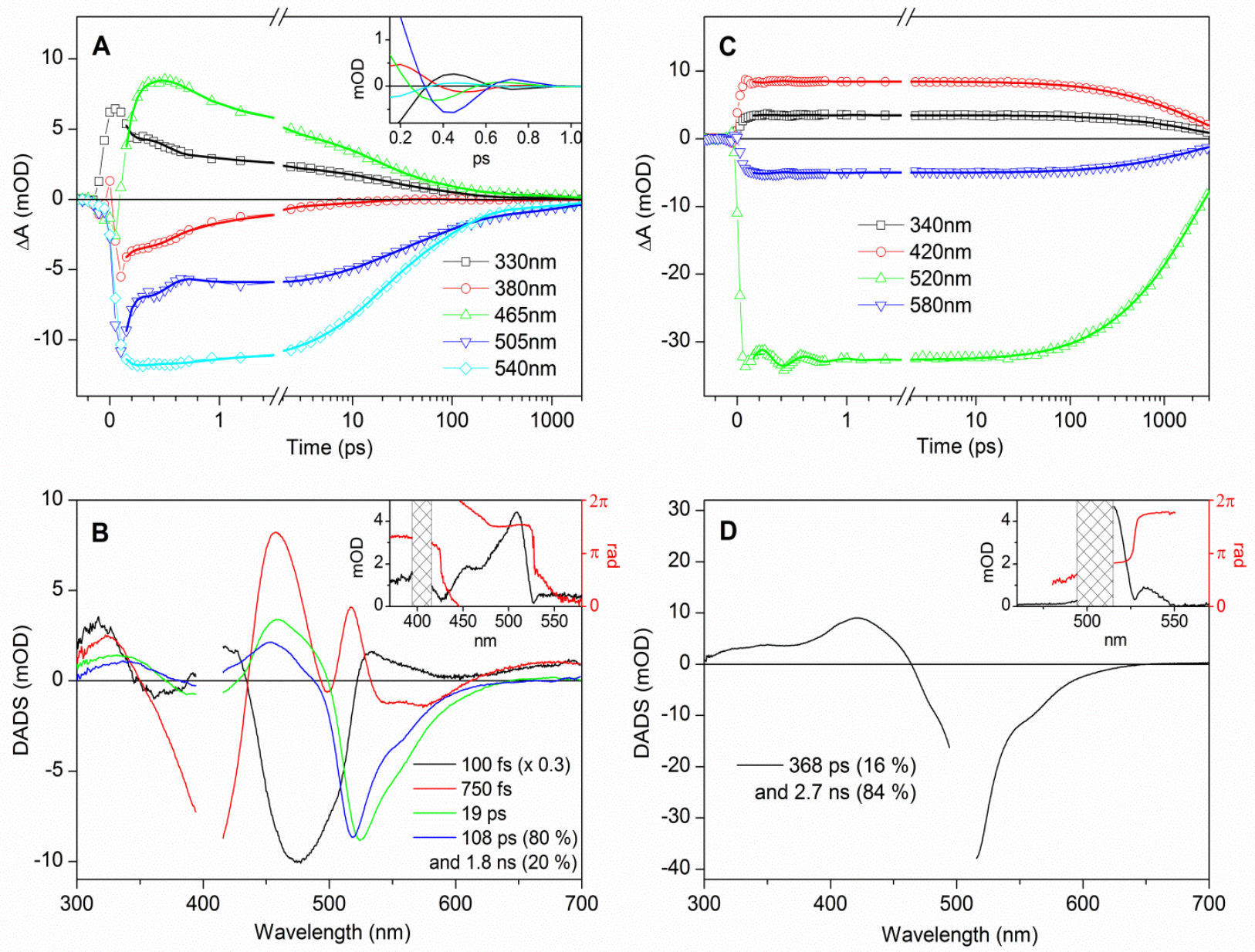


\section{Figure captions}

Figure 1: Steady-state absorption spectrum of Dreiklang in the equilibrium ON state (black) and after 410-nm irradiation (red). The spectra were measured at $\mathrm{pH}$ 7.5.

Figure 2: Femtosecond transient absorption spectra of Dreiklang's neutral ON state (form A) measured at $\mathrm{pH} 6.3$, after 405-nm excitation. The data were corrected for the chirp of the probe. The region near $405 \mathrm{~nm}$, obscured by pump scattering, was masked. (A) 0.1 ps to $0.5 \mathrm{ps}$. The gray dotted, dashed and dash-dotted lines are respectively the steady-state absorption spectrum of the sample, the fluorescence spectrum of the $A^{*}$ state of GFP (emission of the neutral chromophore measured before excited-state deprotonation, by time-resolved fluorescence spectroscopy; taken from Ref. 21) and the steady-state fluorescence spectrum of form B of Dreiklang. The inset shows the double difference spectrum $\Delta \Delta \mathrm{A}=\Delta \mathrm{A}(500 \mathrm{fs})-\Delta \mathrm{A}(100 \mathrm{fs})$ (black line) and its simulation by a weighted difference of neutral and anionic stimulated emissions (red line). (B) 0.5 ps to 3 ps. The inset shows the reshaping of the excited-state absorption on this time-scale, revealed by subtracting the contribution of ground-state bleaching. (C) 3 ps to 2578 ps. The inset displays an additional spectrum measured on the same sample at 10 ns, after complete excited-state decay.

Figure 3: Femtosecond transient absorption spectra of Dreiklang's anionic ON state (form B) measured at $\mathrm{pH} 10.0$, after 505-nm excitation. The data were corrected for the chirp of the probe. The region near $505 \mathrm{~nm}$, obscured by pump scattering, was masked. Inset: Transient absorption spectrum of the same sample at $20 \mathrm{~ns}$.

Figure 4: Global fits of Dreiklang's femtosecond transient absorption data. (A) and (B): Neutral $\mathrm{ON}$ state (form A) at $\mathrm{pH} 6.3$, excited at $405 \mathrm{~nm}$. (C) and (D): Anionic ON state (form B) at $\mathrm{pH}$ 10.0, excited at $505 \mathrm{~nm}$. (A) and (C): Kinetic traces at a few representative wavelengths. The symbols show the measured data points and the thick lines the global fits with a damped cosine 
component and sums of exponentials. Delays shorter than 150 fs are contaminated by the crossphase modulation artifact and were therefore not fitted (see Methods in SI for more details). Inset of section A: Oscillatory part of the fit for form A at the representative wavelengths. The color code is the same as in the main frame. (B) and (D): Decay-associated difference spectra (DADS) of the exponential components. Insets: Amplitude (in black) and phase (in red) spectra of the damped cosine components. The regions near $405 \mathrm{~nm}$ and $505 \mathrm{~nm}$, contaminated by pump scattering, were masked. 
Scheme 1: Chromophore Structures in the ON and OFF States of Dreiklang.

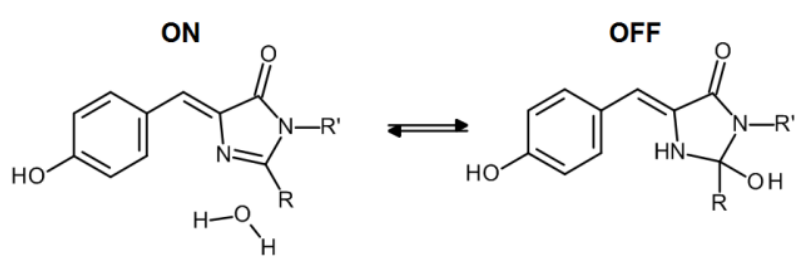


Scheme 2: Proposed Mechanism for the $\mathrm{ON} \rightarrow \mathrm{OFF}$ Photoconversion of Dreiklang. (A) Schematic Excited and Ground-State Potential Energy Surfaces Showing the Intermediate States and Associated Lifetimes. Top : Contour Diagram of the Excited-State Surface Illustrating the Coupling between the Coherently Excited Low-Frequency Vibration and the Reaction Coordinate. (B) Chromophore Hydrogen-Bond Network in the ON State. The Arrows Indicate the 3-Step Rearrangements Assumed to Occur Following Electronic Excitation.
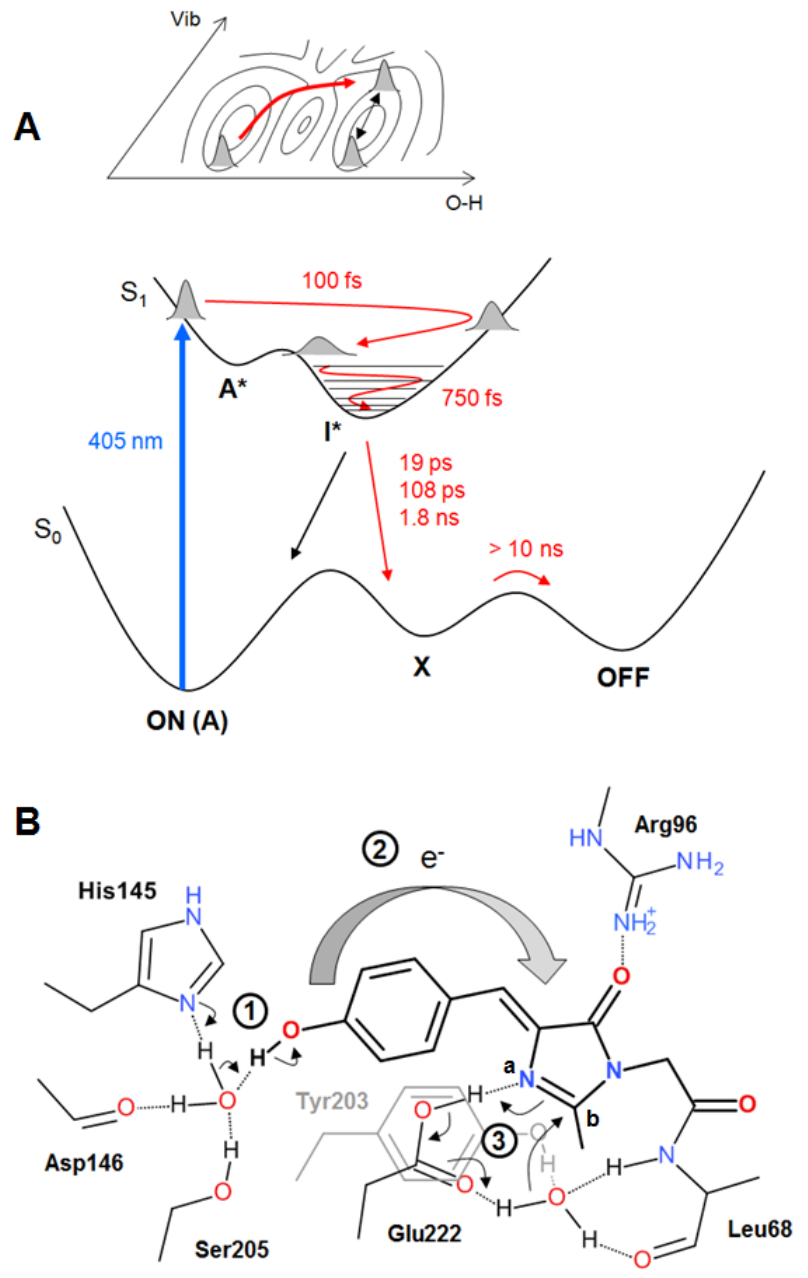